\title{
INTRAOPERATIVE IDENTIFICATION OF SPINAL CORD BLOOD SUPPLY DURING REPAIRS OF DESCENDING AORTA AND THORACOABDOMINAL AORTA
}

Lars G. Svensson, MD, PhD

\begin{abstract}
Objective: The aim was to intraoperatively identify the spinal cord blood supply and shorten the aortic crossclamp time. Methods: A platinum electrode was placed intrathecally by lumbar puncture alongside the spinal cord. After the aorta was crossclamped, hydrogen in a saline solution was injected into the aorta and, if it was shown that the segment supplied the spinal cord and there were multiple arteries, then these were individually injected. The repair was performed by a sequential segmental method as described previously. Results: Postoperatively, highly selective angiography was used to confirm that reattached intercostal arteries supplied the spinal cord. The technique was accurrate in all patients. Five spinal cord perfusion patterns were noted: (1) direct, (2) collateral, (3) no direct supply from segment tested, (4) from atriofemoral bypass, and (5) occluded reattached intercostals. When no response was obtained or no further testing was required $(n=8)$, testing time was 4.2 minutes and crossclamp time 41.9 minutes. When multiple segmental arteries required further testing, the mean testing time was 10.4 minutes and crossclamp time $\mathbf{5 8 . 5}$ minutes, including reattachment of intercostal vessels $(p=$ not significant $)$. Conclusion: Preliminary findings indicate that this method is a safe research technique, can detect radicular arteries, and may reduce the time for aortic crossclamping if no vessels are identified as supplying the spinal cord. (J Thorac Cardiovase Surg 1996;112:1455-61)
\end{abstract}

$\mathrm{R}$ epairs of the descending thoracic aorta and thoracoabdominal aorta have become much safer over time with a concomitant reduction in the mortality rate. ${ }^{1-14}$ Paralysis of the legs has become less of a problem, although paralysis does still develop in some patients, particularly if the aortic crossclamp time is too long or if distal perfusion techniques are not used and segmental intercostal or lumbar arteries are not, or cannot, be reattached

From the Center for Aortic Surgery and the Department of Thoracic and Cardiovascular Surgery, Lahey-Hitchcock Clinic, Burlington, Mass.

Read at the Seventy-sixth Annual Meeting of The American Association for Thoracic Surgery, San Diego, Calif., April 28-May 1, 1996.

Received for publication May 6, 1996; revisions requested July 1, 1966; revisions received August 5, 1996; accepted for publication August 8, 1996.

Address for reprints: Lars G. Svensson, MD, PhD, Director, Center for Aortic Surgery, Department of Thoracic and Cardiovascular Surgery, Lahey Hitchcock Clinic, 41 Mall Rd., Burlington, MA 01805.

Copyright (C) 1996 by Mosby-Year Book, Inc.

$0022-5223 / 96 \$ 5.00+0 \quad \mathbf{1 2 / 6 / 7 7 1 7 9}$ at the time of the operation. Opinions differ on how best to manage intercostal and lumbar arteries at the time of aortic repairs. ${ }^{1-14}$ The concern is that if intercostal and lumbar arteries are reattached to the new tube graft, the duration of aortic crossclamping will be lengthened; because aortic crossclamping is the most significant predictor of the risk of paraplegia or paraparesis, the time required to reattach intercostal vessels may increase the risk of paralysis. Different approaches have been advocated to deal with this problem: reattachment of none of the intercostal or lumbar arteries, to shorten the period of aortic crossclamping ${ }^{14}$; reattachment of the vessels most likely to supply the artery of Adamkiewicz on the basis of anatomic studies, which my colleagues and $\mathrm{I}^{3,4,6,8}$ have advocated by reattaching the intercostals from T-7 down to and including L-1; or the reattachment of all intercostal arteries in the segment of the aorta that is being replaced. ${ }^{10}$ Preoperative highly selective angiography has been used to identify those vessels that supply the spinal cord, but because this method may require a general anesthetic and has resulted in paralysis of the legs in some patients, it is infrequently used. ${ }^{11,15}$ Further- 
more, intraoperatively reconciling the preoperative radiographs with the exact vertebral level of the radicular artery that supplies the spinal cord can be difficult. In a prospective study of patients undergoing operations for type I and type II high-risk thoracoabdominal aneurysms, my colleagues and $\mathrm{I}^{8}$ have shown that patients who did not have intercostal vessels reattached in the segment between T-10 and including L-1 were at a significantly increased risk of postoperative neurologic deficits if these vessels were not reattached at the time of the operation. This clearly indicated that reattachment of patent intercostals is important in preventing paralysis. Reattaching all the patent intercostal vessels, however, would increase the length of time required to perform the operative procedure. We, therefore, concluded that if a method could be found for quickly identifying the vessels supplying the spinal cord, then vessels not supplying the spinal cord could be quickly oversewn, thus potentially shortening the length of aortic crossclamping, and those shown to supply the spinal cord could be reattached.

In porcine animal experiments, we ${ }^{16,17}$ developed a technique in which a catheter with a platinum electrode was placed alongside the spinal cord through a laminectomy. Using the polygraphic method, we injected hydrogen in solution into the aorta, and thus hydrogen reaching the spinal cord was detected by the platinum electrode by producing a current indicating that the intercostal or lumbar artery that had been injected supplied the spinal cord with blood. The preliminary study showed that this was an accurate method of identifying the vessels supplying the spinal cord in the porcine model based on autopsies performed after the experiments. ${ }^{16}$ In a subsequent porcine study, $\mathrm{we}^{17}$ showed that by identifying the vessel supplying the spinal cord and preserving those supplying the spinal cord, but dividing all the other vessels, we could enable animals to recover from the aortic crossclamping without the development of deficits. By contrast, when the vessels identified as supplying the spinal cord were divided, significantly larger numbers of animals became paralyzed. On the basis of these findings, we ${ }^{17}$ considered that this might be a useful technique in human beings. In this study, a series of 14 patients is reported who underwent the procedure, and the results of using this method of identifying the spinal cord blood supply are detailed.

\section{Methods}

Institutional review board approval was obtained from Baylor College of Medicine, the Methodist Hospital, and
Veterans Administration Medical Center in Houston, Texas, and the Lahey-Hitchcock Clinic, Burlington, Massachusetts, for conducting the study. Before the operation, selected patients who were candidates for descending or thoracoabdominal aneurysm repairs were approached and the procedure was fully discussed. Those patients who consented to the procedure were then entered into the study.

After a local anesthetic had been given, a lumbar puncture needle was used to place the previously prepared catheter with the platinum electrode intrathecally. ${ }^{17}$ The platinum wire was then connected for the polygraphic technique as described previously.

Once the operative field had been prepared and an atriofemoral bypass pump had been inserted as needed, the aorta was crossclamped to exclude the segment that was to be repaired. In addition, if an extensive segment of the aorta was to be replaced, then multiple clamps were placed on the aneurysm so that individual segments could be tested. If there was no response from any of these segments, no further testing was performed other than injecting hydrogen also through the atriofemoral bypass machine as it was restarted. In accordance with our approach of doing sequential segmental repairs, the distal clamps were then removed so that only the proximal one third to one quarter of the descending thoracic aorta was clamped, with the rest of the distal aorta being perfused by atriofemoral bypass. The aorta was then transected immediately beyond the left subclavian artery and the proximal anastomosis was performed. If no vessels had been shown to perfuse the spinal cord, the remaining aorta was then crossclamped, the intercostal or lumbar arteries were oversewn, and the graft was sewn in position. When, however, one of the segments was shown to perfuse the spinal cord, then the segment was carefully examined. If more than two pairs of intercostals were patent, then the individual intercostals were injected with hydrogen to try to identify which vessels supplied the spinal cord. Those vessels that were identified as supplying the spinal cord were reattached to the new graft. Before the final clamps were removed to perform the distal aortic anastomoses, patches of reattached intercostal vessels were again tested with hydrogen to ensure that the patches had been successfully reattached and hydrogen was reaching the spinal cord.

After the operation, the patients were checked daily for lower limb function by asking them to raise a straight leg, and the motor responses were then graded according to the grading system $\mathrm{w}^{3-6,8}$ previously described: no movement of the lower limbs, grade 0; a flick of movement, grade 1; ability to bend the knee but not lift the leg, 2; inability to straight-leg raise, with weakness, grade 3 ; and normal leg function with straight-leg raising, grade 4. Once the patients had recovered from the operation and were approaching time of discharge, those who had successful reattachment of intercostal patches underwent highly selective angiography with injection of the intercostal vessels that had been reattached to show if these intercostals supplied the radicular arteries to the spinal cord. Thus we would be able to ascertain the true positive results. False negative results clearly cannot be determined because this would require an autopsy. The length 
of time required for testing and the aortic crossclamp time for the patients who had no response and those who had a response to the hydrogen that required further testing were compared by the Student's $t$ test.

Five patients had descending aortic repairs, three had type I thoracoabdominal aneurysm repairs, and six had type II repairs. There were eleven male patients and three female patients, and the average age was 62.4 years. Seven patients had preoperative aortic dissection. Atriofemoral bypass was used in eight patients, one of whom was converted to full cardiopulmonary bypass, and one other patient had cardiopulmonary bypass from the start of the procedure. Both patients who had cardiopulmonary bypass also underwent circulatory arrest for distal aortic arch repairs.

\section{Results}

The average aortic crossclamp time was 46.6 minutes (range 20 to 85 minutes), and average time for testing was 4.46 minutes (range 0 to 15 minutes). The average time for testing in the patients $(n=7)$ who had no response to injection was 4.5 minutes. With the addition of the one patient who had a response to hydrogen being injected into the atriofemoral bypass circuit without any response in the excluded segment of aorta, the average time was 4.2 minutes. In the latter patient, because the blood vessel supplying the spinal cord was clearly below the segment of occluded aorta, none of the intercostals in the aortic segment needed to be replaced. In the patients with no response, the average crossclamp time was 43.3 minutes, and including the patient who required no further testing, the average crossclamp time was 41.9 minutes. In those patients $(n=4$, Table I) who had a positive response to hydrogen in the segments that were occluded and who required further injection of intercostal arteries, the average time for testing was 10.4 minutes $(p=0.12)$, with an average crossclamp time of 58.5 minutes $(p=0.09)$. The reason for the longer testing and crossclamp times in the latter was that in some patients, multiple intercostal vessels in the segments had to be tested to identify which vessels supplied the spinal cord and this resulted in a longer crossclamp time. Furthermore, these vessels then had to be reattached to the new aortic graft.

In one patient, the preoperative x-ray films showed that the platinum wire within the catheter to the electrode had broken; therefore, the testing would not work. In one other patient, the aneurysm ruptured when the aorta was mobilized and the segments thus could not be excluded for testing. In five patients, patches of the aorta with intercostal vessels were reattached to the new graft. In one
Table I. Hydrogen responses and segmental artery spinal cord blood supply

\begin{tabular}{cll} 
No. & \multicolumn{1}{c}{ Intraop. } & \multicolumn{1}{c}{ Aortogram } \\
\hline 1 & T-10 & Right T-10 ARM \\
2 & T-11 & T-11 collaterals* \\
3 & T-8, T-9, T-10 & Clotted \\
4 & Below T-8 & Left T-9 ARM and T-10 \\
5 & T-9, T-10 & Died \\
\hline
\end{tabular}

$T$, Thoracic vertebral level; $A R M$, arterial radicularis magna; $A F B$, atriofemoral bypass.

*Transient paraparesis.

other patient, the graft was beveled so that the vessels identified as supplying the spinal cord would be preserved without the vessels needing to be reattached separately. Patients who did not have vessels reattached were not studied with highly selective angiography in the postoperative period, because the segmental arteries had been oversewn and excluded from the aortic bloodstream. In one patient, postoperative angiography revealed that the reattached intercostals of the patch had all clotted off at some time after the operation. In one patient in whom a patch had been reattached because of concern that the intercostals were large and could potentially supply the spinal cord, although there had been no good response to hydrogen testing, postoperative highly selective angiography revealed there were no intercostals supplying the spinal cord radicular arteries, confirming that the hydrogen testing had been accurate.

From the hydrogen injection studies and the postoperative highly selective angiography, five spinal cord perfusion patterns were noted:

1. The spinal cord was directly supplied by intercostal vessels via the radicular arteries.

2. Intercostal arteries had been reattached that via collaterals supplied intercostal arteries at higher or lower levels. These latter intercostals were occluded at the ostia from the aorta by atheroma, and these occluded intercostals had originally given rise to the radicular arteries supplying the spinal cord.

3. There was no direct blood supply to the spinal cord from the intercostals tested in the segment that was occluded.

4. The blood supply to the spinal cord arose from the lumbar or lower thoracic intercostal arteries that arose below the level of the occluded segment of the aorta, as detected by the injection of hydrogen into the atriofemoral bypass circuit. 
Table II. Neurologic outcome according to testing

\begin{tabular}{lccc}
\hline & \multicolumn{3}{c}{ Test } \\
\cline { 2 - 4 } & Positive & Negative & Nonet \\
\hline Paraparesis & $1 / 4^{*}$ & $2 / 8 \dagger$ & $0 / 2$ \\
Neurologic score on discharge & 4 & $1 ; 4$ & - \\
\hline
\end{tabular}

Negative test results include one patient in whom blood supply to the spinal cord was below the level that needed to be repaired.

*Transient paraparesis probably caused by blood supply via collaterals.

†One transient paraparesis probably from ruptured aorta and hypotension; one permanent paraparesis from 15 minutes of cardiopulmonary resuscitation for complete heart block.

‡One broken platinum wire; one ruptured aneurysm.

5. The reattached intercostals had clotted off or become occluded after reattachment in the postoperative period.

In no patients were segmental arteries found proximal to the repair; however, the proximal descending aorta was included in the repair in most patients. Clearly, for more distal repairs, proximal thoracic radicular arteries would potentially be present. ${ }^{16-18}$

Permanent paresis (score $=1$ ) developed in one patient $(1 / 14,7 \%)$ after intraoperative cardiopulmonary resuscitation for 15 minutes, necessitated by complete heart block (Table II). The patient was known preoperatively to have left and right bundle branch block as well as chronic atrial fibrillation. $\mathrm{He}$ had required hemodialysis for many years after undergoing a ruptured infrarenal aneurysm repair and renal artery bypass. He successfully underwent an ascending and arch repair for DeBakey type I aortic dissection a few weeks before the thoracoabdominal aneurysm repair. Two other patients had temporary paresis. One was a patient who had previously had an ascending arch repair with an elephant trunk procedure and was undergoing a second-stage elephant trunk procedure for type II thoracoabdominal aneurysm. The patient's hydrogen studies identified the intercostals at T-11 as supplying the spinal cord, and postoperative angiography revealed that the intercostal at $\mathrm{T}-11 \mathrm{had}$ been successfully reattached. However, the blood supply to the spinal cord involved collateral blood supply to the radicular arteries above and below the T-11 vessel, and this may have been a factor in causing transient spinal cord ischemia (score $=3$ ). The patient recovered fully (score $=4$ ). One patient in whom temporary paresis developed was scheduled to have the aorta replaced distally from the subclavian artery for a type II thoracoabdominal aortic dissection. Shortly after the chest was opened, car- diac decompensation occurred and necessitated urgent cardiopulmonary bypass and circulatory arrest to repair the distal aortic arch, which had ruptured. Intraoperatively, no vessels were identified as supplying the spinal cord and, therefore, none were reattached. The patient's postoperative leg weakness was scored as 3 , and by the time of discharge from the hospital, the patient was walking without a problem (score $=4$ ). One patient who required a distal arch repair for a type I thoracoabdominal aneurysm repair was switched from atriofemoral bypass to cardiopulmonary bypass, required circulatory arrest, never awoke, and died shortly afterward. His limb function was unknown.

Thus, of the eight patients in whom no vessels in the segment to be repaired were identified as supplying the spinal cord and in whom, therefore, no vessels needed reattachment, two patients had postoperative paraparesis-one transient (Table II). In the four patients who had vessels localized as supplying the spinal cord and in whom the vessels were reattached, one had postoperative paraparesis. The paraparesis was transient and was probably related to the blood supply to the spinal cord being via collaterals rather than direct.

\section{Discussion}

The prevention of paralysis after aortic crossclamping remains an elusive goal. Methods of reducing the degree and duration of spinal cord ischemia during aortic crossclamping, including atriofemoral bypass, hypothermia, sequential segmental repairs, intrathecal catheters, and pharmacologic agents have been reported previously. ${ }^{1-23}$ From previous studies my colleagues and $\mathrm{I}^{8}$ have reported, successful reattachment of segmental intercostal or lumbar arteries from T-7 to L-1 also is important in reducing the risk of paralysis. Other studies have used somatosensory evoked potentials or motor evoked potentials in attempting to identify segmental arteries that supply the spinal cord. ${ }^{20,22,23}$ $\mathrm{We}^{16,19,24}$ have previously shown that motor evoked responses are very accurate in evaluating spinal cord function and ischemia. This study evaluated in human beings whether an intraoperative method of segmental artery evaluation would determine which segmental arteries supply the spinal cord with blood via the radicular arteries. Using this technique, we observed that the radicular arteries originated below $\mathrm{T}-7$ to and including $\mathrm{T}-11$ in this series of patients.

This study showed that the use of hydrogen in solution and a platinum electrode alongside the 
spinal cord can identify blood vessels that supply the spinal cord, as in our previous study of a porcine model. ${ }^{16,17}$ Furthermore, there were no false positive results. Whether all blood vessels supplying the spinal cord in the repaired aortic segment were detected and identified in human patients is unknown and clearly cannot be determined, because only a postmortem anatomic study would determine if arteries had been missed. In the porcine model, only insignificant, very small $(180 \mu \mathrm{m}, 3 / 28)$ radicular arteries were missed. ${ }^{17}$ In this study, segmental arteries that were not identified as supplying the spinal cord were oversewn, and paresis developed in two patients in this group. In both these latter patients, ischemia per se to the spinal cord during aortic crossclamping was the likely cause (one required cardiopulmonary resuscitation; the other was markedly hypotensive and required cardiopulmonary bypass support followed by circulatory arrest). Of interest, in one patient in whom multiple large intercostal arteries were reattached because of concern that they supplied the spinal cord, despite the hydrogen study showing no response, the postoperative highly selective angiography also did not show that any of the segmental arteries supplied the spinal cord, proving the hydrogen study had been accurate for that particular case. Nevertheless, there are technical reasons why hydrogen testing may fail. These include breakage of the electrode (as happened in one patient), the electrode not being correctly sensitized for hydrogen, the problem of baseline shift with the polargraphic method, the sensitivity of the cables and ammeters to interference and artifact; intrathecal injections or irrigation interfering with recordings, and need for a person to diligently monitor the equipment. ${ }^{16,17,19}$

The method appears to be accurate in determining whether segmental arteries supply the spinal cord according to this study and previous porcine experiments, provided there are no technical problems. The next question is whether the technique can shorten the aortic crossclamp time. If the hydrogen study shows no blood vessels supply the spinal cord from the occluded segment to be repaired, then all the segmental arteries can be oversewn, shortening the crossclamp time, because a patch of segmental arteries does not need to be reattached. In this study, in the patients in whom segmental arteries could be oversewn, the time cost was an average of 4.2 minutes for testing resulting in a total clamp time of 41.9 minutes. Thus approximately 5 to 15 minutes were saved by not having to reattach segmental blood vessels. However, if hydrogen testing showed that blood vessels in the segment to be repaired supplied blood to the spinal cord, then an average of 10.4 minutes (maximum 15 minutes) was required to determine individually which blood vessels supplied the spinal cord, for a total of crossclamp time of 58.5 minutes. This longer crossclamp time reflects not only the longer time for additional testing of each potential artery ostium, but also the time for reattaching segmental arteries that supplied the spinal cord. When hydrogen testing shows blood vessels in the occluded segment supply the spinal cord, an alternative could have been not to have done any further testing to save approximately 6 minutes of additional testing, but reattaching all segmental arteries from $\mathrm{T}-7$ to $\mathrm{L}-1$ based on our previously reported reattachment of likely segmental arteries supplying the spinal cord. ${ }^{6,8,16-18}$ The postoperative angiograms show this latter method would have resulted in the correct segmental arteries being reattached. Of note, based on this series of patients, four of 12 patients (one third) required reattachment of segmental arteries (one additional patient had arteries below the repaired segment supplying the spinal cord). This study confirms our previous recommendations based on autopsy studies that segmental arteries between T-7 and L-1 should be reattached. Of interest, in comparison to our autopsy study of young male trauma victims with normal aortas, ${ }^{18}$ patients with aneurysms or dissection more frequently have occluded segmental ostia and, therefore, have an indirect collateral spinal cord blood supply, either from higher or lower segmental arteries, or from sources that cannot be identified. These sources, however, may be tenuous, as noted on arteriography in one patient in whom transient paresis developed.

In conclusion, this research method is safe and may shorten the crossclamp time for patients who do not require reattachment of segmental arteries. For patients who required reattachment of intercostal arteries, the time cost in identifying these patients, and the segmental arteries that supply the spinal cord, was an average of 10.4 minutes. This latter time could be further shortened by not testing individual segmental artery ostia.

\section{REFERENCES}

1. Crawford ES, Palamara AE, Saleh SA, Roehm JOF. Aortic aneurysm: current status of surgical treatment. Surg Clin North Am 1979;59:597-636. 
2. Crawford ES, Crawford JL, Safi HJ, et al. Thoracoabdominal aortic aneurysms: preoperative and intraoperative factors determining immediate and long-term results of operation in 605 patients. J Vasc Surg 1986;3:389-404.

3. Crawford ES, Svensson LG, Hess KR, et al. A prospective randomized study of cerebrospinal fluid drainage to prevent paraplegia after high-risk surgery on the thoracoabdominal aorta. J Vasc Surg 1991;13:36-45. .

4. Svensson LG, Crawford ES, Coselli JS, Safi HJ. Dissection of the aorta and dissecting aortic aneurysms: improving early and long-term results. Circulation 1990;82(5 Suppl):IV24-38.

5. Svensson LG, Crawford ES, Hess KR, Coselli JS, Safi HJ. Variables predictive of outcome in 832 patients undergoing repairs of the descending thoracic aorta. Chest 1993;104: 1248-53.

6. Svensson LG, Crawford ES, Hess KR, Coselli JS, Safi HJ. Experience with 1509 patients undergoing thoracoabdominal aortic operations. J Vasc Surg 1993;17:357-70.

7. Svensson LG, Shahian DM, Davis FG, et al. Replacement of the entire aorta from the aortic valve to the bifurcation during one operation. Ann Thorac Surg 1994;58:1164-6.

8. Svensson LG, Hess KR, Coselli JS, Safi HJ. Influence of segmental arteries, extent, and atrio-femoral bypass on postoperative paraplegia after thoracoabdominal aortic aneurysm repairs. J Vasc Surg 1994;20:255-62.

9. Coselli JS. Thoracoabdominal aortic aneurysms: experience with 372. J Card Surg 1994;9:638-47.

10. Hollier LH, Money SR, Naslund TC, et al. Risk of spinal cord dysfunction in patients undergoing thoracoabdominal aortic replacement. Am J Surg 1992;164:210-4.

11. Kieffer E, Richard T, Chiras J, Godet G, Cormier E. Preoperative spinal cord arteriography in aneurysmal disease of the descending thoracic and thoracoabdominal aorta: preliminary results in 45 patients. Ann Vasc Surg 1989;3:34-46.

12. Kouchoukos NT, Daily BD, Rokkas CK, et al. Hypothermic bypass and circulatory arrest for operations on the descending thoracic and thoracoabdominal aorta. Ann Thorac Surg 1995;60:67-77.

13. Safi HJ. Neurologic deficit in patients at high risk with thoracoabdominal aortic aneurysms: the role of cerebral spinal fluid drainage and distal aortic perfusion. J Vasc Surg 1994;20:434-43.

14. Acher CW, Wynn MM, Hoch JR, Popic PM, Turnipseed WD. Combined use of spinal fluid drainage and naloxone reduces risk of neurologic deficit in the repair of thoracoabdominal aneurysms. J Vasc Surg 1994;19:236-48

15. Williams GM, Perler BA, Burdick JF, et al. Angiographic localization of spinal cord blood supply and its relationship to postoperative paraplegia. J Vasc Surg 1991;13:23-33.

16. Svensson LG, Patel V, Robinson MF, Ueda T, Roehm JO Jr, Crawford ES. Influence of preservation or perfusion of intraoperatively identified spinal cord blood supply on spinal motor evoked potentials and paraplegia after aortic surgery. J Vasc Surg 1991;13:355-65.

17. Svensson LG, Patel V, Coselli JS, Crawford ES. Preliminary report of localization of spinal cord blood supply by hydrogen during aortic operations. Ann Thorac Surg 1990;49:528-35.

18. Svensson LG, Klepp P, Hinder RA. Spinal cord anatomy of the baboon: comparison with man and implications on spinal cord blood flow during thoracic aortic cross-clamping. S Afr J Surg 1986;24:32-4.

19. Svensson LG, Crawford ES, Patel V, Jones JW, DeBakey
ME. Spinal cord oxygenation, intraoperative blood supply localization, cooling and function with aortic clamping. Ann Thorac Surg 1992;54:74-9.

20. Laschinger JC, Owen J, Rosenbloom M, Cox JL, Kouchoukos NT. Direct noninvasive monitoring of spinal cord motor function during thoracic aortic occlusion: use of motor evoked potentials. J Vasc Surg 1988;7:161-71.

21. Svensson LG, Richards E, Coull A, et al. Relationship of spinal cord blood flow to vascular anatomy during thoracic aortic cross-clamping and shunting. J Thorac Cardiovasc Surg 1986;91:71-8

22. Galla JD, Ergin A, Sadeghi AM, et al. A new technique using somatosensory evoked potential guidance during descending and thoracoabdominal aortic repairs. J Card Surg 1994;9:66272 .

23. Grabitz K, Sandmann W, Stühmeier K, Mainzer B, Godehardt $\mathrm{E}$, et al. The risk of ischemic spinal cord injury in patients undergoing graft replacement for thoracoabdominal aortic aneurysms. J Vasc Surg 1996;23:230-40.

24. Svensson LG, Von Ritter CM, Groenveld HT, et al. Crossclamping of the thoracic aorta: influence of aortic shunts, laminectomy, papaverine, calcium channel blockers, allopurinol, and superoxide dismutase on spinal cord blood flow and paraplegia in baboons. Ann Surg 1986;204:38-47.

\section{Discussion}

Dr. Randall B. Griepp (New York, N.Y.). I would like to recognize the many contributions that Dr. Svensson has made to this field. In many areas we agree and in some we do not. In patients who have atheroma and clot within the aneurysm, what measures have you devised to allow you to multiply clamp the aneurysms without dislodging any of the debris in the vessel lumen?

Dr. Svensson. We have tried to avoid clamping areas where there is atheroma and clot based on the preoperative studies, particularly computed tomographic scans. One of the things that we have done to reduce the risk of embolizing the material is that we have restarted the atriofemoral bypass and then opened the aorta; in other words, flushing the material out proximally. This obviously reduces the danger of flushing the material distally. In one sense, then, we have avoided the problem by evacuating the material. We have not placed any clamps below the level of the visceral vessels and then started atriofemoral bypass, because obviously then we could potentially embolize to the visceral vessels.

Dr. D. Craig Miller (Stanford, Calif.). Let me take that one step further: Even in an atherosclerotic aneurysm and especially in cases of chronic dissection, there usually is extensive clot everywhere. I am surprised that you actually were able to clamp different segmental regions of the aneurysms safely.

Dr. Svensson. That is true. There is quite a lot of clot, and that is a potential risk. I think the danger would be mostly with the clamp squeezing something down the intercostal vessels since we are well above the visceral vessels. With the injection of the hydrogen bolus, if there should be a loose piece of atheroma, I guess there is a possibility of atheroma going down the intercostal artery; however, the ostia of the intercostals usually are much smaller than the pieces of clot that are in the aorta.

Dr. Chris Rokkas (Milwaukee, Wis.). I would like to 
focus on the potential disadvantages of this technique of intraoperative localization of spinal cord blood supply. From your previous publications it appeared that it is not always possible to place those percutaneous platinum electrodes. I wonder if you actually attempted to place those electrodes in patients unsuccessfully. And how big are these electrodes? Can you easily put them through a catheter, or do you actually need to cut down to the vertebral spinal column?

Second, I would like to echo Dr. Griepp's concerns about sequential clamping of the descending aorta because this is not always possible. It can produce embolization of atheromatous debris. How many segments of the aorta do you actually have to crossclamp sequentially before you identify the critical segment? Is that just one, two, three additional crossclampings that are required?

Last, I think the most interesting group of patients is patients with occluded intercostal arteries in the lower thoracic segment and the lumbar segment, patients who actually derive their blood supply through collaterals. Do you think it is important to reimplant the patent upper thoracic intercostal arteries for those patients, although you do not really see a correlation with the hydrogen/ platinum currents?

Dr. Svensson. We were able to insert the catheters with the electrode in every patient without any problems. The size is about $1.8 \mathrm{~mm}$. It is a standard spinal silicone catheter, and the platinum electrode is built onto the catheter on the outside by coiling the platinum wire around the catheter tip. In the patients in whom we used spinal motor evoked potentials, we have either used the platinum electrode or a separate stainless steel electrode on the catheter for stimulation of the spinal cord. The catheter easily goes down a 14-gauge Tuohy needle, and inserting it has not been a problem.

As far as the clamping, in some patients in whom the aneurysms were larger with clot, we did not clamp the aneurysm into individual smaller segments. Instead we opened the aneurysm and injected hydrogen into individual patent intercostal arteries.

As far as what to do with patients who have occluded lower intercostal and lumbar vessels and patent upper intercostal vessels, we have not usually reattached those upper intercostals. However, clearly in patients in whom there are occluded lower vessels, the blood supply is via collaterals. I do not think it is necessarily from the upper thoracic radicular arteries, although those vessels can supply blood flow both upward and downward to the anterior spinal artery. I think many of those patients have collaterals from the intercostal arteries themselves, the internal thoracic, the lateral thoracic, the dorsal scapular, and also the posterior erectae spinae vessels. There are a lot of other potential sources of collateral blood supply to the spinal cord when the intercostals are occluded.

Dr. G. Hossein Almassi (Milwaukee, Wis.). In the single patient in whom the postoperative angiogram revealed that the attached arteries were occluded, I wonder what the outcome was, since presumably the hydrogen study showed that those are the critical arteries that supply blood to the spinal cord. Would you comment on that?

Dr. Svensson. At what stage the patch with the intercostals occluded we do not know, and the patient never had a deficit. It is of interest to us that in patients in whom we do postoperative aortograms, not highly selective aortograms, how often one sees that the patch with the intercostals has clotted off. This is a technical problem that we are going to have to address of how more accurately and safely to reattach the intercostals without them clotting off with time. This may be a factor causing delayed postoperative paraplegia or paraparesis, as our porcine animal research has shown. 